\title{
Design and Simulation of Point of Care Microfluidic Device for Acoustic Blood Cell Separation †
}

\author{
Fatemeh Sharifi ${ }^{1}$, Armin Sedighi ${ }^{2}$ and Mubashar Rehman ${ }^{3, *}$ \\ 1 Sharif University of Technology, Tehran 145888-9694, Iran; Sharifi.fatemeh@gmail.com \\ 2 Science and Research branch, Islamic Azad University, Jahrom 7718184483, Iran; \\ armin.seddighi@gmail.com \\ 3 Quaid-i-Azam University, Islamabad 45320, Pakistan \\ * Correspondence: Mrehman@qau.edu.pk; Tel.: +92-051 90644056 \\ + Presented at the 7th International Electronic Conference on Sensors and Applications, 15-30 November \\ 2020; Available online: https://ecsa-7.sciforum.net/.
}

Published: 15 November 2020

\begin{abstract}
Hematology tests, considered as an initial step in patient diagnostic process, require laboratory equipment and technicians which is a time and labor-consuming procedure. Such facilities may be available in a few central laboratories in under-resourced countries. The growing need for low cost and rapid diagnostic tests contributes to point-of-care (POC) medical diagnostic devices providing convenient and rapid test tools particularly in areas with limited medical resources. In the present study, a comprehensive numerical simulation of POC blood cell separation device (POC-BCS) has been modeled using a finite element method. Tag-less separation of blood cells i.e., platelets, red blood cells, and white blood cells was carried out using standing surface acoustic waves (SSAWs) generated by interdigital transducers (IDTs) located at lateral sides of the microfluidic channel. Blood sample intake along with sheath flow was introduced via two symmetrical tilted angle inlets and middle inlet, respectively. Superposition of acoustic radiation force applied by SSAWs accompanied by drag force caused by medium flow drove the blood cells toward different path lines correlated to their size. White blood cells were sorted out in the middle outlet and, red blood cells and platelets were sorted out through the separate locations of the side outlets. Each cell then guided to their respected visualization chamber for further image processing analysis. The results of the presented numerical study would be very promising in designing and optimizing the POC blood testing device.
\end{abstract}

Keywords: surface acoustic waves; microfluidic devices; point of care devices; blood cell separation

\section{Introduction}

An accurate and expeditious hematology test is considerate as an indispensable step in many diagnostic approaches [1]. The traditional blood test usually requires central laboratories with sophisticated equipment and trained personnel's which may not effortlessly accessible in underresourced countries [1,2]. The recent development of microfluidics and point-of-care (POC) diagnostic devices provide immediate on-site in vitro blood tests showing remarkable potential capabilities in overcoming the mentioned challenges even in deprived areas [1,2].

Many of the prevalent hematology tests require fractionated blood cells [3]. Ultrasound-based Tag-less separation of blood cells exerts an acoustophoretic force on cells and push them based on their size [3]. Such acoustic separation has gained so much attention pertaining to its simplicity, biocompatibility, and effectiveness of this method [1,3,4]. Despite numerous significant experimental studies related to POC blood cell separation (POC-BCS) devices, a lack of detailed numerical studies still exists where different dominant parameters controlling blood cell separation are scrutinized Proceedings 2020, 4, x; doi: FOR PEER REVIEW www.mdpi.com/journal/proceedings 
which in turn leading to an optimized status of the design of the device and its working conditions. In this study, a finite element model of acoustophoretic flow assisted blood cell separation is presented. The overall configuration of the blood cell separation process using the POC blood cell separation device (POC-BCS) is illustrated in Figure 1. The blood sample is taken from the patient and transferred to the POC-BCS device. The blood samples then are directed to the side channels of the POC-BCS device by two connecting tubes. The sheath medium is also circulated through the middle channel. The blood samples and the sheath medium are put inside the syringe pumps so that it pushes both fluids inside the channel. Two interdigital transducers (IDTs) are located at the lateral sides of the POC-BCS device. Standing surface acoustic waves (SSAWs) are generated by these IDTs. SSAWs exert an acoustic radiation force on the particles and pushes them towards specific locations inside the microfluidic channel. The geometry of the device is composed of three inlets, two symmetrical tilted angles inlets, and one middle inlet. The schematic view of the POC-BCS device including dimensions of the different parts is given in Figure 1.

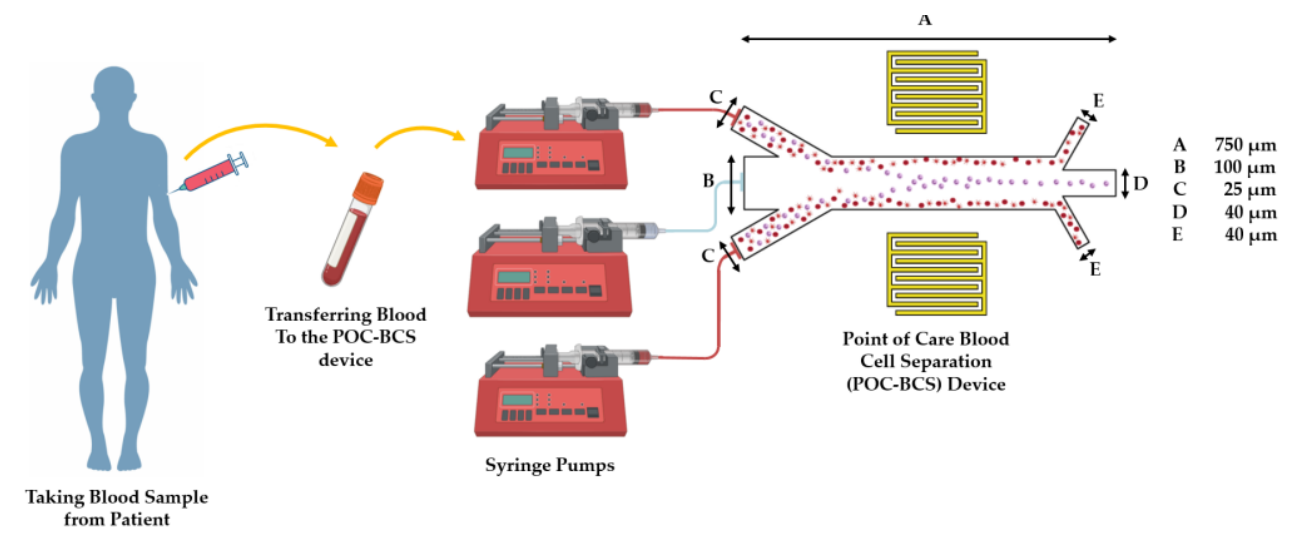

Figure 1. General configuration of the cell separation process. The blood sample is taken from the patient and transferred to the POC-BCS device. The blood samples and sheath media are connected to the POC-BCS device and the circulating force needed to run both media in the channel of the POCBCS device is provided by syringe pumps. Blood enters from two side channels and the sheath media passes through the middle inlet channel. Two IDTs are placed at the top and bottom of the channel providing SSAWs. The SSAWs impose acoustic radiation on the particles and cause them to separate.

\section{Material and Methods}

In the following section, the methodology applied for modeling of POC-BCS device is illustrated. The modeling is composed of three different physics i.e., acoustic pressure fluid flow and particle tracing. The overall process of the presented simulation is depicted in Figure 2. The numerical modeling starts with acquiring acoustic wave propagation by solving the Helmholtz equation where wave propagation and acoustic radiation force are obtained. Then, Navier-Stokes equations are solved so that the velocity distribution of the fluid flow inside the bioreactor is calculated and finally, the exact path of each particle is obtained by solving the equations of motion for each particle. The solution of Helmholtz, continuity, hydrodynamic, and particle tracing equations are attained using 2D finite element methods. The Galerkin method was used in order to approximate the non-linear partial differential governing equations and An Lagrangian approach was chosen to study timedependent particle trajectories. All simulation was carried out on a computer with Corei7 CPU and 12 GB RAM.

\subsection{Governing Equations}

It is presumed that the bioreactor is aligned horizontally and therefore the effects of gravity force are negligible. 


\subsubsection{Wave Propagation}

In this study, the acoustic pressure wave distribution generated by IDTs is calculated by solving Helmholtz equation,

$$
\nabla^{2} p=\frac{\omega^{2}}{c_{f}^{2}} p,
$$

where $p$ is the amplitude of acoustic pressure, $\omega$ is the angular frequency, and $c_{f}$ is the speed of sound in the fluid [5]. Incoming velocity magnitudes induced by acoustic pressure is acquired via;

$$
\boldsymbol{u}_{\text {in }}=-\frac{\nabla p}{\omega \times \rho_{f}}
$$

where $\rho_{f}$ denotes the density of the fluid [5].

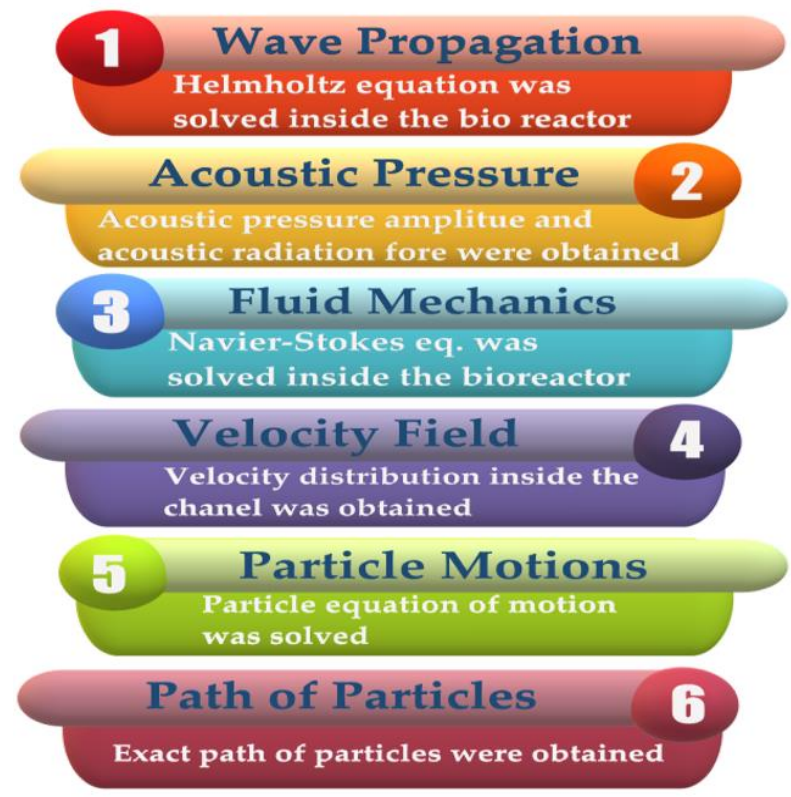

Figure 2. Diagram of the modeling process. First, the Helmholtz equation is solved in order to obtain the wave propagation, acoustic pressure amplitude inside the channel. Next, the acoustic radiation force is calculated using the calculated acoustic pressure. Equations of continuity and momentum are solved subsequently, and the fluid velocity distribution inside the channel is obtained. In the last step, the equation of motion for each particle is solved and the exact path of the particles is obtained.

\subsubsection{Fluid Mechanics}

Velocity distribution is obtained by solving the Navier-Stokes equations. Assuming incompressible, isothermal, Newtonian fluid, the equations of continuity and momentum can be written as

$$
\begin{gathered}
\nabla \cdot \boldsymbol{u}=0, \\
\rho_{f}\left(\frac{\partial \boldsymbol{u}}{\partial t}+\boldsymbol{u} \cdot \nabla \cdot \boldsymbol{u}\right)=\nabla \cdot\left[-p \boldsymbol{I}+\mu\left(\nabla \boldsymbol{u}+(\nabla \boldsymbol{u})^{\boldsymbol{T}}\right)\right]
\end{gathered}
$$

where $u, \mu$ are fluid velocity and viscosity, respectively [6]. As previously mentioned, the channel is assumed to be horizontally, and therefore, the effect of the gravity force is neglected. Since the perfusion rate is low, the Reynolds number indicating the ratio of inertial forces to viscose forces are small $(\operatorname{Re}<2000)$ which in turn results in a laminar flow regime inside the channel [6].

\subsubsection{Particle Motions}

Once the acoustic pressure and velocity distribution field is calculated, it is possible to determine the forces applied on a single suspended particle. When the acoustic pressure propagates through a 
medium containing particles, it exerts an acoustic radiation force stemming from a scattering of incident SSAWs, $\boldsymbol{F}_{\text {rad }}$, on these particles. By assuming blood particles to be spherical, $\boldsymbol{F}_{\text {rad }}$ can be computed as

$$
\begin{gathered}
\boldsymbol{F}_{\text {rad }}=-\frac{4 \pi}{3} a^{3}\left(\frac{f_{1}}{2 c_{f}^{2} \rho_{f}} \overline{p^{2}}-\frac{3 f_{2}}{4} \rho_{f} \overline{u_{l n}^{2}}\right), \\
f_{1}=1-\frac{\kappa_{s}}{\kappa_{f}} \text { and } f_{2}=2 \frac{\left(\rho_{p}-\rho_{f}\right)}{2 \rho_{p}+\rho_{f}}
\end{gathered}
$$

where $\overline{p^{2}}, \overline{u_{l n}^{2}}$ and $\rho_{p}$ indicate mean square of pressure, incoming velocity, and particle density, respectively. $\kappa$ denotes compressibility [5]. Acoustic radiation force pushes the particles towards the pressure nodes and pressure antinodes based on the sign of acoustic factors, i.e., $f_{1}$ and $f_{2}$.

Particles in the fluid also experience another force called Stokes drag force, which also depends on the relative motion of the particles respect to flow. When a particle with radius a moves at the velocity of $\boldsymbol{u}_{p}$ inside the fluid with the flow rate of $\boldsymbol{u}_{f}$, then the Stokes drag force is computed as:

$$
\boldsymbol{F}_{\text {drag }}=-6 \pi \mu a\left(\boldsymbol{u}_{p}-\boldsymbol{u}_{f}\right)
$$

By writing the Newton second law, force balance, for each particle, the particle trajectory can be obtained as:

$$
\boldsymbol{F}_{\text {drag }}+\boldsymbol{F}_{\text {rad }}=m \frac{d^{2} \boldsymbol{r}}{d t^{2}}
$$

where $m$ and $r$ describe mass and displacement of the particle [4].

\subsection{Boundary Conditions}

Boundary conditions for SSAWs are considered as a prescribed wall motion in y-direction for modeling the effect of IDTs,

$$
\boldsymbol{u}_{i n, y}=A * \cos (\omega t)
$$

where $A$ is velocity amplitude. The other walls, shown with blue color in Figure 3a, are modeled as a hard wall, so

$$
\boldsymbol{u}_{\text {in }}=0,
$$

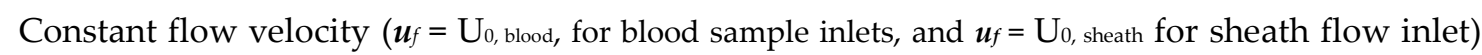
and outflow boundary condition ( $\mathrm{\partial} u / \partial \boldsymbol{n}=0, n$ is a normal vector) at the three outlets of the fluid flow channel are considered as the inlet and outlet boundary conditions, respectively (Figure 3b). It also assumed that fluid flow will be fully developed at three outlets. No-slip (zero velocity) boundary condition is applied on the other walls.

Particles enter the channel with constant velocity $\left(\boldsymbol{u}_{p}=\mathrm{U}_{0}\right.$, blood). As the particles pass through the channel, they experience two forces mentioned earlier, i.e., acoustic radiation force and drag forces. At the outlet, particle velocity is $\boldsymbol{u}_{p}=u_{p}$ which is considered the velocity of the particle when striking the wall (Figure 3c). Parameters and constants used in this simulation are given in Table 1.

Table 1. Parameters and constants used in the simulation.

\begin{tabular}{ccc}
\hline Simulation Parameter-Symbol [SI Unite] & Value & Source \\
\hline water viscosity $-\mu[\mathrm{Pa}-\mathrm{s}]$ & 0.001 & {$[6]$} \\
water density $-\rho_{f}\left[\mathrm{~kg} / \mathrm{m}^{3}\right]$ & 1000 & {$[6]$} \\
speed of sound in water $-c_{f}[\mathrm{~m} / \mathrm{s}]$ & 1481 & {$[5]$} \\
compressibility $-\kappa[1 / \mathrm{Pa}]$ & $4 \times 10^{-10}$ & {$[5]$} \\
Acoustic velocity amplitude $-A[\mathrm{~nm} / \mathrm{s}]$ & 5 & calculated \\
Angular frequency $-\omega[\mathrm{rad} / \mathrm{s}]$ & $30 \times 10^{6}$ & calculated \\
Sheath flow velocity at inlet $-\mathrm{U}_{0, \text { sheath }}[\mathrm{m} / \mathrm{s}]$ & $2.5 \times 10^{-2}$ & calculated \\
Blood velocity at inlet $-\mathrm{U}_{0, \text { blood }}[\mathrm{m} / \mathrm{s}]$ & $10^{-2}$ & calculated \\
\hline
\end{tabular}



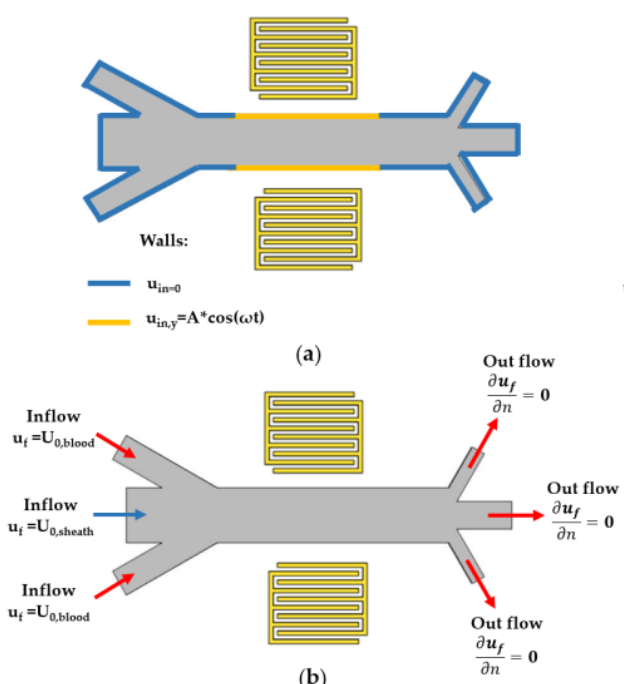

(b)
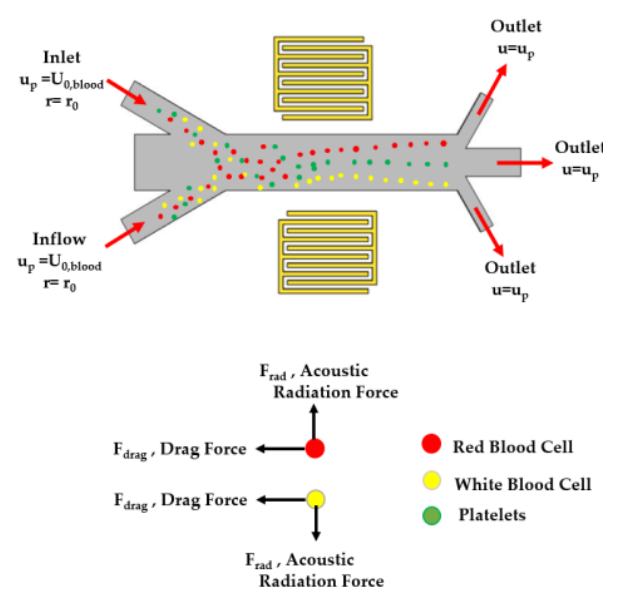

(c)

Figure 3. The imposed boundary condition used for current simulation. (a) Boundary conditions used for modeling of SSAWs; (b) Boundary Conditions applied for the modeling of fluid flow; (c) Boundary conditions exploited for simulation of particle motion. Scales are plotted larger for the sake of clarity.

\section{Results}

Fluid velocity distribution along the width of the channel is plotted for different mesh number (Figure 4a), providing mesh size independency of the results from the numerical simulation. It can be inferred from Figure 4a that grid with 4172 elements are sufficient for further analysis and finer grid numbers do not show any significant discrepancies.

It is important to find an optimum conditions among dominant parameters affecting the particle separation, so that blood cell separate efficiently. Therefore, parameters like angular frequency, channel dimensions, and velocity amplitude were chosen so that the blood cells separate inside the POC-BCS device. Fluid velocity distribution in the channel along with streamlines are illustrated in Figure $4 \mathrm{~b}$. Since the Reynolds number is small $(\operatorname{Re}=6)$, the fluid flow is laminar and the parabolic pattern of the flow across the channel width is obvious. Figure 4c exhibits the acoustic pressure distribution inside the channel. Acoustic pressure is generated at the walls beside the IDTs, therefore, in the down part of the microchannel, the acoustic pressure amplitude becomes zero. The angular frequency was chosen so that the pressure node is formed in the middle part of the channel which in turn leading to an appropriate separation process.

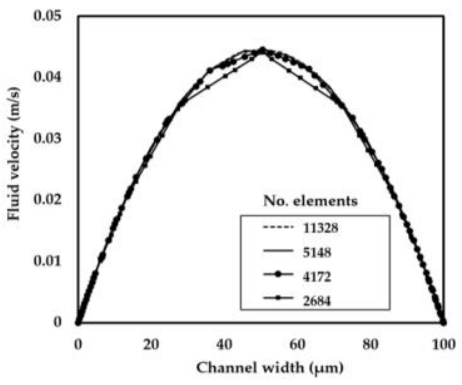

(a)

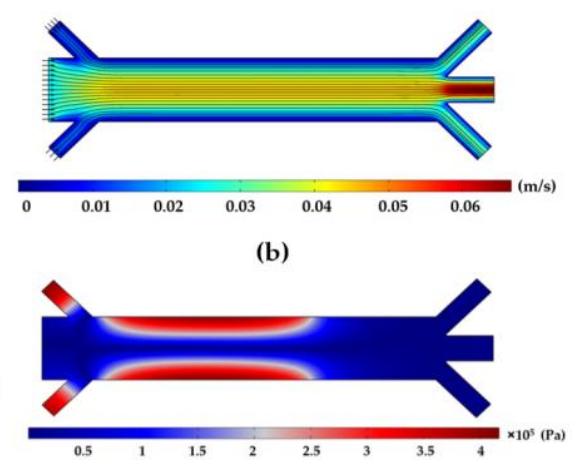

(c)

Figure 4. Results of grid independency, velocity, and acoustic pressure distribution. (a) Profile of velocity variation along the width of the microfluidic channel for different element size; (b) Fluid velocity distribution along with streamlines inside the POC-BCS channel; (c) Acoustic pressure amplitude distribution at the angular frequency of $30 \mathrm{rad} / \mathrm{s}$. 
Particle trajectories for red blood cells (red circles, diameter $8 \mu \mathrm{m}$ ), white blood cells (yellow circles, diameter $14 \mu \mathrm{m}$ ), and platelets (green circles, diameter $2 \mu \mathrm{m}$ ) inside the channel are shown in Figure 5. As previously mentioned, particles experience two forces, drag, and acoustic radiation force, along their path to the outlets. The magnitude of the acoustic radiation force is proportional to the cube of particle diameter (Equation (5)), therefore, larger acoustic radiation force applies to bigger particles and it is expected that the particles with the bigger diameter will be pushed towards the centerline of the microchannel. Blood cell trajectories at three different time points are depicted in Figure 5a-c. It can be seen that the white blood cells are gathered at the middle outlet and red blood cells and platelets are sorting out through separate locations of the side outlets.

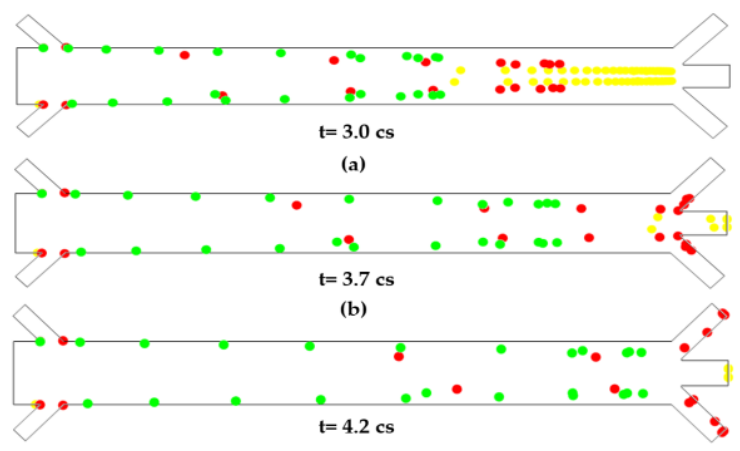

(c)

Figure 5. Particle trajectories inside the channel at different time. (a) $t=3.0 \mathrm{cs}$; (b) $t=3.7 \mathrm{cs}$; (c) $t=4.7 \mathrm{cs}$.

\section{Discussions and Conclusions}

In the presented study a two-dimensional POC-BCS device simulation is analyzed in which blood cells are sorted using SSAWs. The modeling is developed based on a finite element simulation. Helmholtz equation, Navier-Stokes equations are solved along with the equation of motion for the modeling of blood cell separation. The angular frequency of $30 \times 10^{6} \mathrm{rad} / \mathrm{s}$ and blood inlet velocity of $0.01 \mathrm{~m} / \mathrm{s}$ was found to be the optimum condition for the separation. The peak of the acoustic pressure is located beside the IDTs. It can be observed that the white blood cells are gathered at the middle outlet and red blood cells and platelets are sorting out through separate locations of the side outlets. Hence, using the acquired parameters, the blood cells can be separated without any cell markers.

Author Contributions: F.S. and A.S. conceived of the presented idea. They developed the theory and performed the computations. She developed the theoretical formalism, performed the analytic calculations, and performed the numerical simulations. M.R. verified the analytical methods and supervised the findings of this work. F.S. wrote the manuscript under the supervision of M.R. All authors discussed the results and contributed to the final manuscript.

Conflicts of Interest: “The authors declare no conflict of interest."

\section{References}

1. Li, H.; Steckl, A.J. Paper microfluidics for point-of-care blood-based analysis and diagnostics. Anal. Chem. 2018, 91, 352-371.

2. Ellett, F.; Jorgensen, J.; Marand, A.L.; Liu, Y.M.; Martinez, M.M.; Sein, V.; Butler, K.L.; Lee, J.; Irimia, D. Diagnosis of sepsis from a drop of blood by measurement of spontaneous neutrophil motility in a microfluidic assay. Nat. Biomed. Eng. 2018, 2, 207-214.

3. Yousuff, C.M.; Ho, E.T.W.; Hussain, K., I.; Hamid, N.H.B. Microfluidic platform for cell isolation and manipulation based on cell properties. Micromachines 2017, 8, 15.

4. Wu, M.; Ozcelik, A.; Rufo, J.; Wang, Z.; Fang, R.; Huang, T.J. Acoustofluidic separation of cells and particles. Microsyst. Nanoeng. 2019, 5, 32.

5. Franke, T.; Hoppe, R.; Linsenmann, C.; Zeleke, K. Numerical simulation of surface acoustic wave actuated cell sorting. Open Math. 2013, 11, 760-778.

6. White, F.M.; Corfield, I. Viscous Fluid Flow; McGraw-Hill: New York, NY, USA, 2006; Volume 3. 
Publisher's Note: MDPI stays neutral with regard to jurisdictional claims in published maps and institutional affiliations.

(C) 2020 by the authors. Submitted for possible open access publication under the terms and conditions of the Creative Commons Attribution (CC BY) license (http://creativecommons.org/licenses/by/4.0/). 